\title{
Rigid Molecule Approximation in Memory Function-based Models for Molecular Liquids: Application to Liquid Water
}

\author{
By Vania Calandrini ${ }^{1}$, Godehard Sutmann ${ }^{2}$, Antonio Deriu ${ }^{3}$, and \\ Gerald Reinhard Kneller ${ }^{1, *}$ \\ 1 Centre de Biophysique Moléculaire, CNRS, Rue Charles Sadron, Orléans 45071, France \\ 2 Jülich Supercomputing Centre, Research Centre Jülich, 52425 Jülich, Germany \\ 3 Physics Department and CNISM, Universitá di Parma, Via G.P. Usberti 7/A, Parma \\ 43100, Italy
}

Dedicated to Prof. Dr. Alfons Geiger on the occasion of his $65^{\text {th }}$ birthday

(Received July 24, 2009; accepted July 27, 2009)

\section{Memory Function-based Models / Molecular Liquids / Liquid Water}

In the present article we show how models for simple liquids can be used to describe the dynamics of atoms in molecular liquids within the rigid molecule approximation. We show in particular that the atomic masses are to be replaced by the corresponding Sachs-Teller masses and we derive a formal expression for the so-called Einstein frequency. The approach is illustrated for a model which has been originally developed for simple liquids and which has been used in the past to analyze quasielastic neutron scattering data from pure water and dilute aqueous solutions of apolar molecules. We obtain a remarkable agreement with results from molecular dynamics simulations not only in the quasielastic, diffusive regime, but also in the inelastic regime corresponding to intermolecular vibrations and fast molecular librations.

\section{Introduction}

A deeper understanding of the dynamics of liquids is still a challenging task and many discoveries are still to be expected, although considerable progress has been undoubtedly made, in particular through molecular dynamics (MD) simulations. In this context the work of Rahman is to be cited in the first place. His pioneering simulation of liquid argon [1] was an important step for the development of models for simple liquids, a survey of which can be found in several

* Corresponding author. E-mail: gerald.kneller@cnrs-orleans.fr 
monographs $[2,3,4]$. Of particular interest for the theory of liquids and complex molecular systems in general is the Generalized Langevin equation, which leads to a rigorous description of the relaxation of time correlation functions on the basis of a projection formalism $[5,6,7,8]$. The so-called memory function is the cornerstone in this context. Although its direct calculation is as unfeasible as the solution of the Hamiltonian equations of motion for a given system, it turns out to be very useful for the development of models for the dynamics of liquids. One of the first memory function-based models for the velocity autocorrelation function of simple liquids [9] showed that even a simple model for the memory function yields a qualitatively correct behavior of the associated time correlation function.

Most of the models for the dynamics of liquids which have been developed in the past concern so-called simple liquids, like liquid argon, the constituents of which are atoms. The use of such models for complex liquids, such as polymers and proteins in solution, does not pose a problem in principle, as long as these systems can be considered as mixtures of simple liquids. This requires the absence of rigid units or sub-units, in which certain atoms are connected through stiff bonds. One might argue that models for molecular liquids involving rigid or semi-rigid molecules should simply not be used to keep the theoretical framework as simple as possible, but the rigid molecule approach is not only a theoretical concept but also a physical reality if one considers, for example, the scattering of thermal neutrons by molecular liquids. If the energy of the neutrons is not sufficient to excite molecular vibrations, the molecules appear as rigid in the scattering process. These considerations lead Sachs and Teller to introduce effective masses for atoms in rigid molecules when they studied the scattering of neutrons by molecular gases [10]. The same situation arises in todays neutron scattering studies of the dynamics of molecular liquids, such as liquid water or ice, where neutrons of moderate energy are used. This is typically the case for quasielastic neutron scattering (QENS) studies, which probe the diffusive dynamics of the molecules. Due to the dominant cross section for incoherent scattering of the hydrogen atoms, one sees effectively the dynamics of a "liquid of hydrogen atoms" in such experiments. To be able to use theoretical models designed for simple liquids for the interpretation of such experiments one needs, for example, the mean square velocity of a hydrogen atom in a rigid molecule. It has been shown by one of us (GRK) that thermal averaging in velocity space in presence of rigid-body constraints leads to the standard expression for the mean square velocity of a particle in a simple liquid if the atomic mass is replaced by the Sachs-Teller mass [11]. This result has been used in a MD simulation study of liquid water [12], in which we applied the concept of effective atomic masses to refine a memory-function based model for the single particle dynamics in liquids, in order to describe the dynamics of the hydrogen atoms in water within the rigid molecule approximation. The original model has been used before by two of us (VC and AD) to interpret QENS experiments on water and aqueous solutions of apolar molecules $[13,14]$. Here we review and extend the 
theoretical considerations, giving also a formal expression for the so-called Einstein frequency of atoms in liquids consisting of rigid or semi-rigid molecules, and, compared to [12], we present supplementary simulation results and an improved method for the calculation of higher order memory functions.

The article is organized as follows: In Section 2 we present the theoretical background and Section 3 contains short description of the simulation protocol, as well as a presentation of the numerical calculation of memory functions from MD simulations. The results are discussed in Section 4 and the paper is concluded by a short summary of the results.

\section{Theoretical background}

\subsection{Generalized Langevin equation and memory functions}

It has been shown in a very general way $[5,6,8]$ that the equation of motion for a given dynamical variable $A(t)$ can be written as

$$
\dot{A}(t)=i \omega_{A} A(t)-\int_{0}^{t} d \tau M_{A}(t-\tau) A(\tau)+f_{A}(t),
$$

where $\omega_{A}$ is a proper frequency, $f_{A}(t)$ is a "projected force", and $M_{A}(t)=$ $\left\langle f_{A}(0) f_{A}(t)\right\rangle\langle A A\rangle^{-1}$ is the memory function. The projected force has the fundamental property $\left\langle f_{A}(t) A(0)\right\rangle=0$, which allows deriving a closed equation for the autocorrelation function $c_{A A}(t)=\left\langle A^{*}(0) A(t)\right\rangle$,

$$
\dot{c}_{A A}(t)=i \omega_{A} c_{A A}(t)-\int_{0}^{t} d \tau M_{A}(t-\tau) c_{A A}(\tau) .
$$

If one assumes that $c_{A A}(t)$ is a classical autocorrelation function, as we do in this article, it follows that $c_{A A}(t)=c_{A A}(-t)$ and consequently $\dot{c}_{A A}(0)=i \omega_{A}=0$. We may thus set $\omega_{A}=0$ from now on. The Laplace transform of $c_{A A}(t)$, defined as $\hat{c}_{A A}(s)=\int_{0}{ }^{t} d t \exp (-s t) c_{A A}(t)(\Re\{s\}>0)$, takes the particularly simple algebraic form

$$
\hat{c}_{A A}(s)=\frac{c_{A A}(0)}{s+\hat{M}_{A}(s)}
$$

which may be used to set up a hierarchical scheme by introducing higher order memory functions. The idea is to treat the projected force $f_{A}(t)$ in a way entirely analogous to the one established for $A(t)$, defining second-order fluctuating forces and memory functions. The result is similar to Eq. (3), with $\hat{c}_{A A}(s)$ replaced by $\hat{M}_{A}(s)$, and with $\omega_{A}$ and $\hat{M}_{A}(s)$ replaced by their second-order counterparts. Substituting this result for $\hat{M}_{A}(s)$ into Eq. (3) and iterating the procedure to arbitrary order one finally gets a representation of $\hat{c}_{A A}(s)$ in form of a continued fraction, as derived by Mori [7]. 


$$
\hat{c}_{A A}(s)=\frac{c_{A A}(0)}{s+\frac{M_{A}^{(1)}(0)}{s+\frac{M_{A}^{(2)}(0)}{s+\ldots}}} .
$$

Here $M^{(n)}(0)$ denotes the initial value of the $n$-th order memory function.

\subsection{A model for the dynamics of liquids}

In the following we consider the intermediate scattering function for single particle motion,

$$
F_{s}(q, t)=\langle\exp (i q[x(t)-x(0)])\rangle,
$$

where $F_{s}(q, t) \equiv c_{A A}(t), A(t)=\exp (i q x(t)), x$ is a Cartesian component of the position of a tagged scattering atom, and $q$ is the momentum transfer from the neutron to the sample. Here we suppose that the system is isotropic, which implies that all directions in space are equivalent. The dynamics of $F_{s}(q, t)$ is described by a second order Mori-Ansatz, such that

$$
\hat{F}_{s}(q, s)=\frac{1}{s+\frac{M^{(1)}(q, 0)}{s+\hat{M}^{(2)}(q, s)}},
$$

with $F_{s}(q, 0)=1$. The initial values for the memory functions are related to the Taylor coefficients for the short time expansion of $F_{s}(q, t)$ ("sum rules") [2,4], and one may write

$$
\begin{aligned}
& M^{(1)}(q, 0)=\left\langle v^{2}\right\rangle q^{2}, \\
& M^{(2)}(q, 0)=2\left\langle v^{2}\right\rangle q^{2}+\Omega^{2} .
\end{aligned}
$$

Here $v=\dot{x}$ is the time derivative of the Cartesian position coordinate $x$ appearing in the intermediate scattering function (5), and $\Omega$ is the "Einstein frequency",

$$
\Omega^{2}=\frac{\left\langle\dot{v}^{2}\right\rangle}{\left\langle v^{2}\right\rangle} .
$$

We note that $\Omega^{2}$ is the initial value of the memory function associated with the velocity autocorrelation function (VACF), $c_{v v}(t)=\langle v(t) v(0)\rangle$. Writing

$$
\dot{c}_{v v}(t)=-\int_{0}^{t} d \tau M_{v}^{(1)}(t-\tau) c_{v v}(\tau)
$$

we have

$$
\Omega^{2}=M_{v}^{(1)}(0) .
$$

The choice of a second order Mori ansatz for $F_{s}(q, t)$ can be motivated by the equation of motion corresponding to relation (6),

$$
\ddot{F}_{s}(q, t)+\int_{0}^{t} d \tau M^{(2)}(q, \tau) \dot{F}_{s}(q, t-\tau)+q^{2}\left\langle v^{2}\right\rangle F_{s}(q, t)=0 .
$$


Setting $M^{(2)}(q, t)=\tau(q)^{-1} \delta(t)$, Eq. (12) takes the form of the equation of motion of a damped harmonic oscillator, with a relaxation constant $\gamma(q)=\tau(q)^{-1}$ and an angular frequency $q^{2}\left\langle v^{2}\right\rangle$, describing the short-time dynamics of $F_{s}(q, t)$.

For the second order memory function a model including two relaxation processes has been put forward, which has been used in particular for the interpretation of incoherent quasi-elastic neutron scattering from pure water $[15,16]$ and from dilute aqueous solutions of apolar molecules [17,13,14],

$$
M^{(2)}(q, t)=M^{(2)}(q, 0)\left\{\alpha \exp \left(-t / \tau_{1}\right)+(1-\alpha) \exp \left(-t / \tau_{2}\right)\right\} .
$$

Here $\tau_{1}$ and $\tau_{2}$ are the characteristic times concerning "fast" relaxation processes due to intermolecular collisions and "slow" structural relaxation processes, respectively, and the parameter $\alpha$ verifies $0 \leq \alpha \leq 1$. The dependence on $q$ of these parameters is here omitted. Using expression (6) together with the initial values of the first and second order memory function, which are given by Eqs. (7) and Eqs. (8), respectively, one obtains the following expression for the Laplace transformed intermediate scattering function:

$$
\hat{F}_{s}(q, s)=\frac{1}{s+\frac{\left\langle v^{2}\right\rangle q^{2}}{s+\left(2\left\langle v^{2}\right\rangle q^{2}+\Omega^{2}\right)\left\{\frac{\alpha}{s+\tau_{1}^{-1}}+\frac{1-\alpha}{s+\tau_{2}^{-1}}\right\}}} .
$$

The above expression leads to a rational function for $\hat{F}_{s}(q, s)$, exhibiting four poles in the $s$-plane, and the intermediate scattering function is thus a weighted sum of four (possibly complex) exponential functions. By construction, such a model will work for a certain time window, which is set by the relaxation times and frequencies obtained from the poles of $\hat{F}_{s}(q, s)$. One must be aware that hydrodynamic long-time tails, which manifest themselves through an algebraic decay of correlation functions in the limit of infinite time, cannot be obtained in a mathematical sense, i.e. in the formal limit $t \rightarrow \infty$.

Eq. (14) is the starting point to derive various quantities which are relevant to neutron scattering. Using that $F_{s}(q, t)$ is symmetric in time, the dynamic structure factor takes the form

$$
S_{s}(q, \omega)=\frac{1}{2 \pi} \int_{-\infty}^{+\infty} d t \exp (-i \omega t) F_{s}(q, t)=\frac{1}{\pi} \mathfrak{R}\left\{\hat{F}_{s}(q, i \omega)\right\} .
$$

The VACF can be obtained from the relation $c_{v v}(t)=-\lim _{q \rightarrow 0} q^{-2} \partial_{t}^{2} F_{s}(q, t)$. With $F_{s}(q, 0)=1$ and $\partial_{t} F_{s}(q, 0)=0$ one obtains for the Laplace transform

$$
\begin{aligned}
\hat{c}_{v v}(s) & =-\lim _{q \rightarrow 0} \frac{1}{q^{2}}\left\{s^{2} \hat{F}_{s}(q, s)-s\right\} \\
& =\frac{\left(s+\tau_{1}^{-1}\right)\left(s+\tau_{2}^{-1}\right)\left\langle v^{2}\right\rangle}{s^{3}+s^{2}\left(\tau_{1}^{-1}+\tau_{2}^{-1}\right)+s\left(\tau_{1}^{-1} \tau_{2}^{-1}\right)+\Omega^{2}\left([1-\alpha] \tau_{1}^{-1}+\alpha \tau_{2}^{-1}\right)} .
\end{aligned}
$$

The above expression yields the Fourier transform of the VACF, known as "density of states" (DOS), via

$$
\tilde{c}_{v v}(\omega)=\int_{-\infty}^{+\infty} d t \exp (-i \omega t) c_{v v}(t)=2 \mathfrak{R}\left\{\hat{c}_{v v}(i \omega)\right\}
$$


From the Kubo relation $D=\int_{0}^{\infty} d t c_{v v}(t)=\hat{c}_{v v}(0)$ one obtains the following expression for the diffusion coefficient of the model,

$$
D=\frac{\left\langle v^{2}\right\rangle}{\Omega^{2}} \frac{1}{\alpha \tau_{1}+(1-\alpha) \tau_{2}} .
$$

Using that the Laplace transformed VACF may be written in the form $\hat{c}_{v v}(s)=$ $\left\langle v^{2}\right\rangle\left(s+\hat{M}^{(1)}{ }_{v}(s)\right)^{-1}$, where $\hat{M}^{(1)}{ }_{v}(s)$ is the Laplace transform of the corresponding first order memory function, it follows that

$$
D=\frac{\left\langle v^{2}\right\rangle}{\gamma_{v}}
$$

where $\gamma_{v}$ is the friction constant, which is defined as

$$
\gamma_{v}=\int_{0}^{\infty} d t M_{v}^{(1)}(t)
$$

Here one can make use of the identity

$$
M_{v}^{(1)}(t)=M^{(2)}(0, t)
$$

which follows from the time derivative of Eq. (12) and from the relation $c_{v v}(t)=$ $-\lim _{q \rightarrow 0} q^{-2} \partial_{t}^{2} F_{s}(q, t)$. With $\dot{F}_{s}(q, 0)=0$ and $\dot{F}_{s}(0, t)=0$, one obtains

$$
\dot{c}_{v v}(t)=-\int_{0}^{t} d \tau M^{(2)}(0, t-\tau) c_{v v}(\tau),
$$

which proves relation (21). Using the general form (13) for the second order memory function of $F_{s}(q, t)$ and that $M^{(2)}(0,0)=\Omega^{2}$ according to relation (8), it follows thus that

$$
M_{v}^{(1)}(t)=\Omega^{2}\left\{\alpha \exp \left(-t / \tau_{1}\right)+(1-\alpha) \exp \left(-t / \tau_{2}\right)\right\} .
$$

The corresponding friction constant is given by

$$
\gamma_{v}=\Omega^{2}\left\{\alpha \tau_{1}+(1-\alpha) \tau_{2}\right\}
$$

and may be inserted into relation (19) to retrieve expression (18) for the diffusion coefficient of the model.

\subsection{Rigid molecule approximation}

\subsubsection{Mean square velocity of a selected atom}

In the model described previously the mean square velocity, $\left\langle v^{2}\right\rangle$, is one of the parameters. We recall that $v$ is actually one Cartesian component of the velocity v. For a simple liquid the calculation of the mean square velocity is straightforwardly obtained from the average $\left\langle v^{2}\right\rangle=\int_{-\infty}^{+\infty} d v v^{2} p(v)$, where $p(v)$ is the Maxwell distribution, $\left.p(v)=\sqrt{[(2 \pi) /(M \beta)]} \exp (-M \beta / 2] v^{2}\right)$, with $\beta=\left(k_{B} T\right)^{-1}, k_{B}$ being the Boltzmann constant, $T$ the absolute temperature, and $M$ the mass of the particle. One obtains the well known result $\left\langle v^{2}\right\rangle=k_{B} T / M$.

Consider now a molecule consisting of $N$ atoms, which are linked by $s$ geometrical constraints with $s \leq 3 N$, 


$$
h_{k}(\mathbf{r})=h_{k}^{(0)}, \quad k=1 \ldots s,
$$

where $h_{k}^{(0)}=$ const. Here $\mathbf{r}$ is a column vector of length $3 N$ which comprises all Cartesian coordinates of the $N$ atoms. We note that rigid body constraints in the form (25), where $s=3 N-6$, may be achieved through a combination of quadratic bond constraints and linear constraints for the atomic positions [18]. Differentiation of (25) yields a set of $s$ constraints for the velocity components of the atoms in the molecule,

$$
\mathbf{A v}=\mathbf{0}
$$

where $\mathbf{v}=\dot{\mathbf{r}}$ and $\mathbf{A}$ is a $s \times 3 N$ dimensional matrix whose elements are given by

$$
A_{k i}=\frac{\partial h_{k}}{\partial r_{i}}, \quad i=1, \ldots, 3 N, \quad k=1, \ldots, s .
$$

Eq. (26) shows that $\mathbf{v}$ is in the null space of $\mathbf{A}$, which will be denoted $V_{\|}$in the following. Its dimension is the number of degrees of freedom, $\operatorname{dim}\left(V_{\mid}\right)=3 \mathrm{~N}$ $\operatorname{rank}(\mathbf{A})$, where $\operatorname{rank}(\mathbf{A}) \leq s$. If all constraints are independent, $\operatorname{rank}(\mathbf{A})=s$. The orthogonal complement of $V_{\|}$is denoted $V_{\perp}$ and $\operatorname{dim}\left(V_{\perp}\right)=\operatorname{rank}(\mathbf{A})$.

It is clear that geometrical constraints will create dependencies among the velocity components. For purposes of thermal averaging one must therefore consider a modified Maxwell distribution involving the velocities of all atoms in the molecule. To construct this distribution function it is convenient to introduce the projectors on $V_{\|}$and $V_{\perp}$, which are given by

$$
\begin{aligned}
& \mathbf{P}_{\|}=\mathbf{A}^{+} \mathbf{A}, \\
& \mathbf{P}_{\perp}=\mathbf{1}-\mathbf{P}_{\|},
\end{aligned}
$$

respectively, where $\mathbf{A}^{+}$denotes the pseudoinverse of $\mathbf{A}[19,20]$. Numerically $\mathbf{A}^{+}$ can be obtained from the singular value decomposition (SVD) [21] of $\mathbf{A}$. If $\mathbf{A}$ has full rank $s$, its pseudoinverse can be given explicitly,

$$
\mathbf{A}^{+}=\mathbf{A}^{T}\left(\mathbf{A} \mathbf{A}^{T}\right)^{-1},
$$

where the superscript " $T$ " denotes a transposition.

With the above prerequisites and following the lines in Ref. [11] (here for the velocities and not for the momenta), the modified Maxwell distribution takes the form

$$
p(\mathbf{v})=\sqrt{\frac{2 \pi}{\beta}} f \frac{\delta\left(\mathbf{P}_{\perp} \mathbf{v}\right) \exp \left(-\frac{\beta}{2} \mathbf{v}^{T} \mathbf{M}_{c} \mathbf{v}\right)}{\sqrt{\operatorname{det}\left(\mathbf{M P} \mathbf{P}_{\|}+\mathbf{P}_{\perp}\right)}},
$$

where $\mathbf{M}_{c}$ is the projection of the diagonal mass matrix,

$$
\mathbf{M}=\left(\begin{array}{cccc}
m_{1} \mathbf{1} & \mathbf{0} & \ldots & \mathbf{0} \\
\mathbf{0} & m_{2} \mathbf{1} & \ldots & \mathbf{0} \\
\vdots & \vdots & \vdots & \vdots \\
\mathbf{0} & \mathbf{0} & \ldots & m_{N} \mathbf{1}
\end{array}\right),
$$


onto the subspace $V_{||}$,

$$
\mathbf{M}_{c}=\mathbf{P}_{\|} \mathbf{M P} \mathbf{P}_{\|},
$$

and the Dirac distribution accounts for the velocity constraint (26) which may be written in the alternative forms $\mathbf{P}_{||} \mathbf{v}=\mathbf{v}$ and $\mathbf{P}_{\perp} \mathbf{v}=\mathbf{0}$. One derives from the constrained Maxwell distribution (31) that [11]

$$
\left\langle\mathbf{v} \mathbf{v}^{T}\right\rangle=k_{B} T \mathbf{M}_{c}^{+} .
$$

In case that $\operatorname{rank}(\mathbf{A})=s$, the matrix $\mathbf{M}_{c}^{+}$can be expressed in the form [11]

$$
\mathbf{M}_{c}^{+}=\mathbf{M}^{-1}-\mathbf{M}^{-1} \mathbf{A}^{T}\left(\mathbf{A} \mathbf{M}^{-1} \mathbf{A}^{T}\right)^{-1} \mathbf{A} \mathbf{M}^{-1} \text {. }
$$

In order to obtain the mean square velocity of individual atoms, the matrix $\mathbf{M}_{c}^{+}$ is partitioned in $3 \times 3$ block matrices,

$$
\mathbf{M}_{c}^{+}=\left(\begin{array}{cccc}
\mathbf{m}_{11}^{+} & \mathbf{m}_{12}^{+} & \ldots & \mathbf{m}_{1 N}^{+} \\
\mathbf{m}_{21}^{+} & \mathbf{m}_{22}^{+} & \ldots & \mathbf{m}_{2 N}^{+} \\
\vdots & \ldots & \ldots & \vdots \\
\mathbf{m}_{N 1}^{+} & \ldots & \ldots & \mathbf{m}_{N N}^{+}
\end{array}\right) .
$$

For a tagged atom $\alpha$ one has thus $\left\langle\mathbf{v}_{\alpha} \mathbf{v}_{\alpha}{ }^{T}\right\rangle=k_{B} T \mathbf{m}_{\alpha \alpha}{ }^{+}$, and it follows that the mean square velocity is given by

$$
\left\langle\mathbf{v}_{\alpha}^{T} \mathbf{v}_{\alpha}\right\rangle=k_{B} T \operatorname{tr}\left\{\mathbf{m}_{\alpha \alpha}^{+}\right\} .
$$

The effective mass $M_{\alpha, \text { eff }}$ can be defined through the relation $\left\langle\mathbf{v}_{\alpha}^{T} \mathbf{v}_{\alpha}\right\rangle=3 k_{B} T /$ $M_{\alpha, \text { eff }}$, leading to

$$
M_{\alpha, \text { eff }}^{-1}=\frac{1}{3} \operatorname{tr}\left\{\mathbf{m}_{\alpha \alpha}^{+}\right\}
$$

Noting that the mean square velocity $\left\langle v^{2}\right\rangle$ appearing in the dynamical model introduced in Section 2.2 concerns the projection of $\mathbf{v}$ onto a unit vector in an arbitrary direction, we have thus

$$
\left\langle v^{2}\right\rangle=\frac{k_{B} T}{M_{\alpha, \text { eff }}} .
$$

\subsubsection{Einstein frequency}

The other basic parameter in the dynamical model for the intermediate scattering function is the Einstein frequency, which is defined by $\Omega^{2}=\left\langle\dot{v}^{2}\right\rangle /\left\langle v^{2}\right\rangle$ and which is the initial value of the memory function associated with the VACF. The derivation of an analytical expression for the Einstein frequency of an atom in a rigid molecule involves thus the calculation of its mean square acceleration in presence of geometrical constraints. Formally, such an expression can be given. For this purpose one derives constraints for the accelerations of the atoms in a (semi)rigid molecule by differentiating relation (26) with respect to time. This yields an expression of the form 


$$
\mathbf{A} \dot{\mathbf{v}}=\mathbf{b},
$$

where the vector $\mathbf{b}$ reads

$$
\mathbf{b}=-\dot{\mathbf{A}} \mathbf{v} \text {. }
$$

Starting from Newton's equations of motion,

$$
\mathbf{M} \dot{\mathbf{v}}=\mathbf{f}+\mathbf{z},
$$

where $\mathbf{f}$ comprises the external forces acting on the atoms in the molecule under consideration and $\mathbf{z}$ are the constraint forces, one can derive an explicit expression for the vector of accelerations [22],

$$
\dot{\mathbf{v}}=\mathbf{M}_{c}^{+} \mathbf{f}+\left(\mathbf{1}-\mathbf{M}_{c}^{+} \mathbf{M}\right) \mathbf{A}^{+} \mathbf{b},
$$

where $\mathbf{A}^{+}$and $\mathbf{M}_{c}^{+}$are given by (30) and (35), respectively. To extract the acceleration of atom $\alpha$ from (43) we introduce the $3 \times 3 N$ matrix $\mathbf{S}_{\alpha}$

$$
\mathbf{S}_{\alpha}=\left(\begin{array}{lllllll}
\mathbf{0} & \ldots, & \mathbf{0}, & \mathbf{1}, & \mathbf{0}, & \ldots, & \mathbf{0}
\end{array}\right),
$$

such that

$$
\dot{\mathbf{v}}_{\alpha}=\mathbf{S}_{\alpha} \dot{\mathbf{v}}
$$

The Einstein frequency of a tagged atom $\alpha$ may then be written as

$$
\Omega^{2}=\frac{\left\langle\dot{\mathbf{v}}_{\alpha}^{T} \dot{\mathbf{v}}_{\alpha}\right\rangle}{\left\langle\mathbf{v}_{\alpha}^{T} \mathbf{v}_{\alpha}\right\rangle}=\frac{M_{\alpha, \text { eff }}}{3 k_{B} T}\left\langle\dot{\mathbf{v}}^{T} \mathbf{S}_{\alpha}^{T} \mathbf{S}_{\alpha} \dot{\mathbf{v}}\right\rangle .
$$

\section{Molecular Dynamics simulation and analysis}

\subsection{MD simulation}

For the present study of the dynamics of liquid water we have performed MD simulations of 256 water molecules in a cubic box of edge length $1.9552 \mathrm{~nm}$ in the thermodynamic $N p T$-ensemble at a temperature of $T=300 \mathrm{~K}$ and a pressure of 1 bar, using the SPC/E potential [23] and Ewald summation for long-range electrostatic interactions. Within the SPC/E model the water molecules are treated as rigid bodies which are composed of point masses, carrying each an electric charge. Only the oxygen atoms carry in addition a center for interactions of Lennard-Jones type. All simulations have been performed with the simulation program DL_POLY (version 2) [24], using a simulation time step of $1 \mathrm{fs}$. In order to be able to study slow diffusive motions of water molecules on one hand, resolving on the other hand at the same time the first order memory function sufficiently well for short times, we used an MD trajectory of $100 \mathrm{ps}$ with a sampling interval of $10 \mathrm{fs}$. For the calculation of second order memory functions we created a short trajectory of $20 \mathrm{ps}$ with a sampling interval of $2 f_{s}$. 


\subsection{Calculation of higher order memory functions}

To calculate higher order memory functions we start from Eq. (2), replacing the autocorrelation function $c(t)$ (the index $A A$ is omitted) by the memory function of order $n-1$ and setting $\omega_{A}=0$,

$$
\dot{M}^{(n-1)}(t)=-\int_{0}^{t} d \tau M^{(n)}(t-\tau) M^{(n-1)}(\tau) .
$$

where $n \geq 1$ and $M^{(0)}(t) \equiv c(t)$. The discretized form of this integro-differential equation reads

$$
\frac{M^{(n-1)}(k+1)-M^{(n-1)}(k)}{\Delta t}=-\Delta t \sum_{j=0}^{k} M^{(n)}(k-j) M^{(n-1)}(j),
$$

where $M(k) \equiv M(k \Delta t)(k \in Z)$. Applying a one-sided z-transform to (2), which is defined through

$$
\hat{M}_{>}(z)=\sum_{k=0}^{\infty} M(k) z^{-k}
$$

one obtains

$$
\hat{M}_{>}^{(n)}(z)=\frac{1}{\Delta t^{2}}\left(\frac{z M^{(n-1)}(0)}{\hat{M}_{>}^{(n-1)}(z)}+1-z\right) .
$$

In the following it is supposed that the memory function $M^{(n-1)}(k)$ is already known. Starting from the first level in this hierachy of calculations, the basic quantity to know is the correlation function $c(t) \equiv M^{(0)}(t)$. To estimate $c(t)$ (and its Fourier spectrum) from MD trajectories we use autoregressive (AR) modeling [25] for the time series of the underlying dynamical variables. The calculation of autocorrelation functions within the AR model is described in Ref. [26].

Using the definition (3) of the z-transform together with Eq. (4), one obtains the relation

$$
\sum_{j=0}^{\infty} M^{(n)}(j) z^{-j}=\frac{1}{\Delta t^{2}} \frac{\sum_{j=0}^{\infty}\left(M^{(n-1)}(j)-M^{(n-1)}(j+1)\right) z^{-j}}{\sum_{j=0}^{\infty} M^{(n-1)}(j) z^{-j}} .
$$

Note that the term proportional to $z$ cancels out. The time dependent memory function is, in principle, obtained by comparing the coefficients of the series on the lhs and the rhs of Eq. (5). To construct a numerical method we replace the series by polynomials of order $N$, where $T=N \Delta t$ defines the time window for the memory function to be computed. After this first step a polynomial division is performed on the rhs of Eq. (5), and after a subsequent multiplication of both sides with $z^{-N}$ one obtains the time dependent memory function, $M^{(n)}(j)$, by comparison of coefficients, 


$$
\begin{aligned}
\frac{z^{-N}}{\Delta t^{2}} \frac{\sum_{j=0}^{N}\left(M^{(n-1)}(j)-M^{(n-1)}(j+1)\right) z^{N-j}}{\sum_{j=0}^{N} M^{(n-1)}(j) z^{-j}} & =z^{-N}\left(\sum_{j=0}^{N} c_{j} z^{N-j}+R\right) \\
& =\sum_{j=0}^{N} M_{j}^{(n)} z^{-j} .
\end{aligned}
$$

The coefficients $c_{j}$ are obtained by polynomial division and $R$ is a rest which does not contain information on the memory function within the time interval $t$ $\in[0, T]$. The discrete memory function is therefore given by $M^{(n)}(j)=c_{N-j}$.

In contrast to the method described in [26], the above scheme for the calculation of memory functions is more stable from a numerical point of view and does not require the use of program packages for calculations with extended precision. It allows, moreover, to obtain memory functions of arbitrary order within the same numerical scheme. The only limitation is the due to the finite resolution of the time correlation function which is used as starting point for the calculations. As mentioned earlier, higher order memory functions decay increasingly rapidly, and one arrives quickly at the point where the time resolution is not fine enough to reconstruct them. If one is interested in memory functions of order $n>3$, one might be forced to use time steps which are smaller than those usually used for MD simulation.

\section{Results and discussion}

\subsection{Dynamics of the hydrogen and oxygen atoms}

In a first step we analyzed separately the dynamics of the oxygen and hydrogen atoms, considering for both types of atoms the respective intermediate scattering function and the associated memory function. Both intermediate scattering functions have been averaged over the respective number of equivalent atoms. The analysis was motivated by the interpretation of quasielastic neutron scattering data in [13], where the analytical model described in Section 2.2 has been used with a point-like representation of the water molecules. Here only the center-ofmass dynamics was considered, which can be approximately identified with the dynamics of the oxygen atoms. Although it is the incoherent scattering from hydrogen which dominates quasielastic neutron scattering spectra, this approach is justified if one is interested in the slow long range diffusive dynamics only, to which the fast relative motions of the hydrogen atoms about the respective centers of mass do not contribute. Fig. 1 illustrates this point. Using a point-like representation of the water molecules yields a good fit of the quasielastic part of the dynamic structure factor at $q=10 \mathrm{~nm}^{-1}$, which deviates, however, considerably from the MD results in the inelastic part of the spectrum. In Ref. [13] the mean square velocity in the model was fixed through $\left\langle v^{2}\right\rangle=k_{B} T / M$, where $M$ 


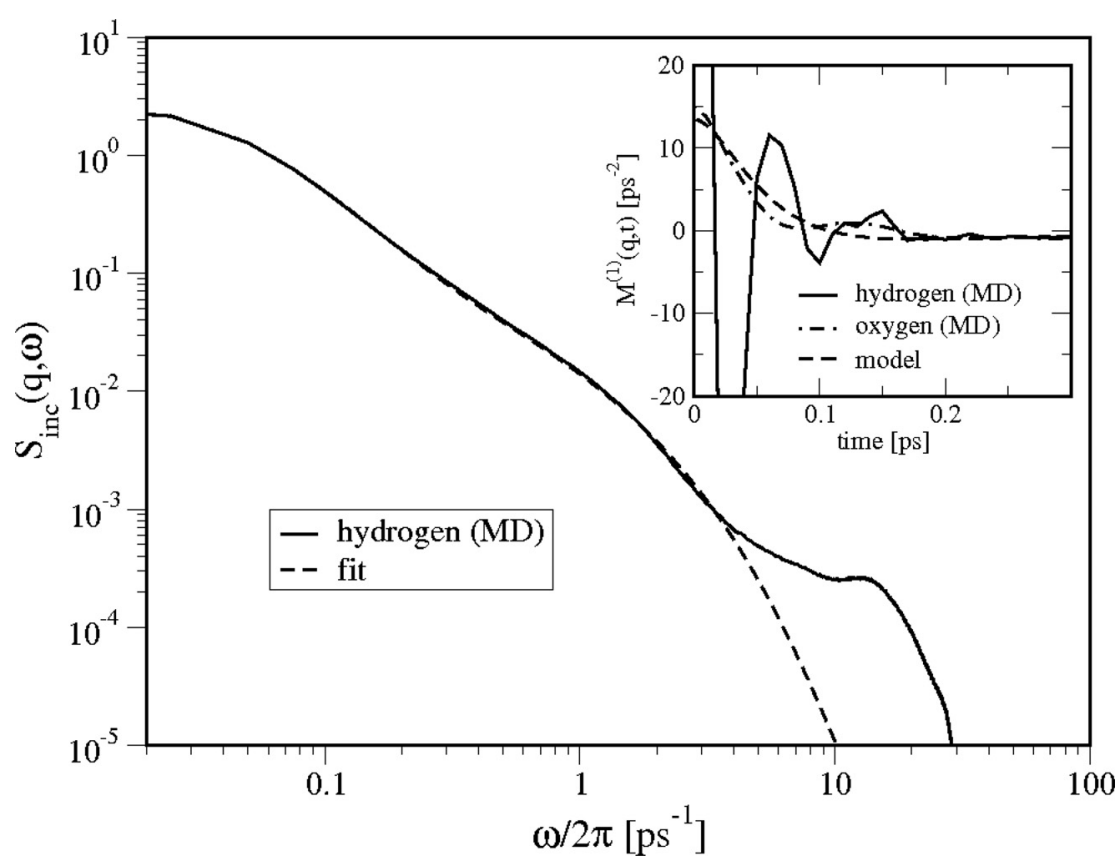

Fig. 1. Incoherent dynamic structure factor from MD simulation at $q=10 \mathrm{~nm}^{-1}$ (solid line) and fit of the analytical model, using the molecular mass as mass of the scattering particle (broken line). The inset shows the first order memory function of the intermediate scattering function for the hydrogen atoms (solid line), the corresponding quantity for the oxygen atoms (broken and dotted line), and first order memory function corresponding to the fitted intermediate scattering function (broken line).

is the molecular mass. The Einstein frequency was treated as a fit parameter, which was initialized by the theoretical value obtained from the expression [4]

$$
\begin{aligned}
\Omega^{2}= & \frac{\left\langle\dot{v}^{2}\right\rangle}{\left\langle v^{2}\right\rangle} \approx \frac{\left\langle\dot{v}_{O}^{2}\right\rangle}{\left\langle v^{2}\right\rangle} \approx \frac{4 \pi n}{3 M} \int_{0}^{\infty} r^{2} d r\left[\frac{\partial^{2} U_{O O}(r)}{\partial r^{2}}+\right. \\
& \left.\frac{2}{r}\left(\frac{\partial U_{O O}(r)}{\partial r}\right)\right] g_{O O}(r) .
\end{aligned}
$$

Here $U_{O O}(r)$ is the oxygen-oxygen potential taken from the SPC/E MD force field, $n$ is the molecular density of water, and $g_{O O}(r)$ is the oxygen-oxygen pair correlation function obtained from MD simulation (not shown here). We note here that (1) involves formally two approximations, (a) the replacement of the center-of-mass by the position of the oxygen atom and (b) the evaluation of the mean square acceleration of the oxygen atom without taking into account the effects of constraint forces. Using a simulated oxygen-oxygen pair correlation function, we (VC and $\mathrm{AD}$ ) obtained 


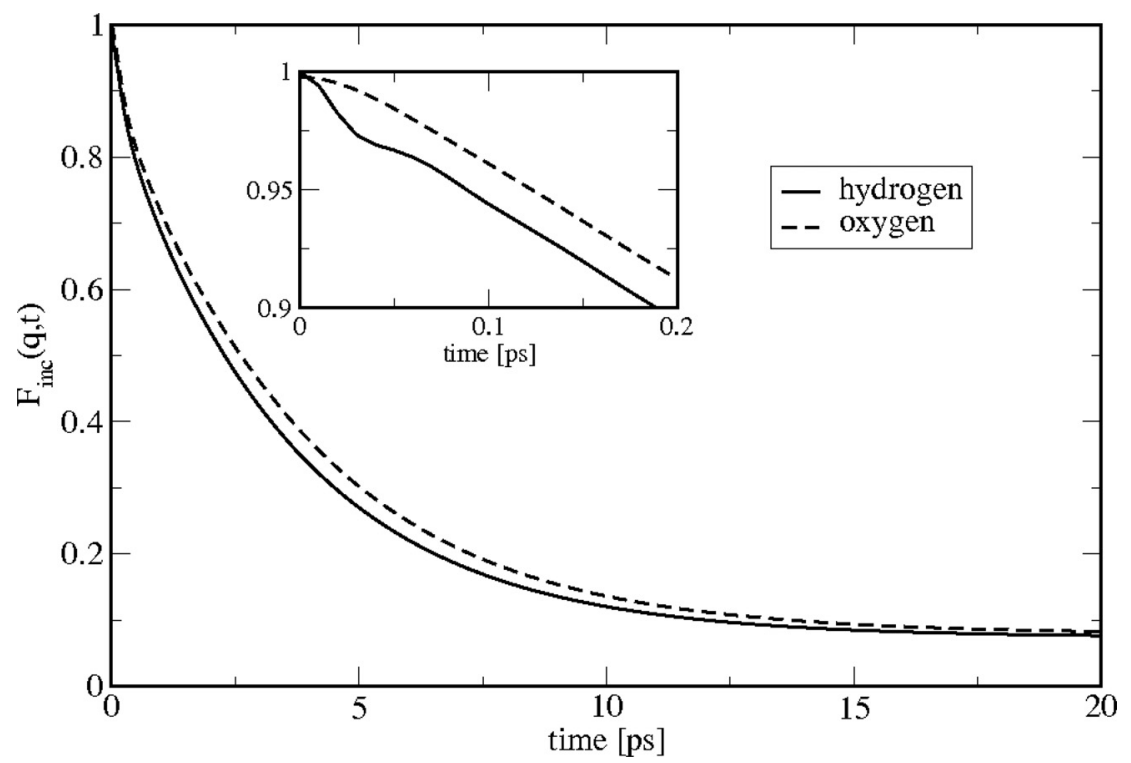

Fig. 2. Incoherent intermediate scattering function for hydrogen (solid line) and oxygen (broken line) at $q=10 \mathrm{~nm}^{-1}$. The inset shows a zoom on the intermediate scattering functions for small times.

$$
\Omega^{2} \approx 1322 p s^{-2}
$$

which is compatible with the quasielastic neutron scattering data.

The inset of Fig. 1 shows that the first order memory function of $F_{s}(q, t)$ for the hydrogen atoms (solid line) and the oxygen atoms (dashed-dotted line) obtained from MD simulation, and the corresponding model memory function obtained from the parameters of the fit (dashed line). One recognizes that the latter agrees for short times much better with the memory function for the oxygen atoms than with the one for the hydrogen atoms. Fig. 2 confirms the above observations in the sense that the long time behavior of the intermediate scattering function is the same for hydrogen as for oxygen. Only the short time behavior is different (see inset). We note here that the simulated intermediate scattering functions have been obtained for $q=10 \mathrm{~nm}^{-1}$, using autoregressive modeling, with 400 poles and a sampling time step of $10 \mathrm{fs}$.

To understand why quite different memory functions can lead to a very similar relaxation behavior of the associated correlation functions we consider the corresponding relaxation or friction constants,

$$
\gamma(q)=\int_{0}^{\infty} d t M^{(1)}(q, t) .
$$

At $q=10 \mathrm{~nm}^{-1}$ (Fig. 2) one finds $\gamma=0.31 \mathrm{ps}^{-1}$ for hydrogen and $\gamma=0.28 \mathrm{ps}^{-1}$ for oxygen. As a rough approximation one may set $F(t) \approx \exp [-\gamma(q) t]$, which 
becomes an exact relation in the regime of Brownian motion. This type of motion is explored in the hydrodynamic limit, $q \rightarrow 0$. In this case one may write

$$
F_{s}(q, t) \approx \exp \left(-D q^{2} t\right),
$$

showing that $\gamma \approx D q^{2}$, where $D$ is the diffusion coefficient. For small but finite values of $q$ (here $q=10 \mathrm{~nm}^{-1}$ ) one obtains thus estimations of the diffusion coefficients for hydrogen and and oxygen: $D_{H} \approx 3.1 \cdot 10^{-5} \mathrm{~cm}^{2} / \mathrm{s}, D_{O} \approx 2.8$. $10^{-5} \mathrm{~cm}^{2} / \mathrm{s}$. These values are slightly above the diffusion coefficient of $D=2.55$ $\cdot 10^{-5} \mathrm{~cm}^{2} / \mathrm{s}$ which is found from the mean square displacement for both hydrogen and oxygen and which is close to the experimental value of $D=2.23 \cdot 10^{-5}$ $\mathrm{cm}^{2} / \mathrm{s}$ found by NMR measurements [27].

\subsection{Modeling the full hydrogen dynamics}

The next question to be asked is if the analytical model introduced above is also able to describe the full dynamics of the hydrogen atoms in water and not only slow diffusive motions, which are described by the oxygen atoms whose positions almost coincide with the center-of-mass. Here not only slow diffusive motions are to be described, but also faster intermolecular vibrations and molecular librations. As already mentioned, the rigid-body representation of a water molecule corresponds to the physical situation encountered in the scattering of thermal neutrons by small molecules in general, since internal vibrations cannot be excited in this case. It is also the basis of the SPC/E model for water which has been used in the simulations. Within the model for $F_{s}(q, t)$, the Einstein frequency, $\Omega$, and the mean square velocity of the scattering atom, $\left\langle v^{2}\right\rangle$, are to be considered as input parameters which can be, in principle, obtained from first principles, according Eqs. (46) and (39). The calculation of the Einstein frequency according to (46) is, however, beyond the scope of this article since it is less straightforward than in the case of simple liquids (compare Expression (1)). For this reason we use the value obtained from MD simulation. From the initial values of the first order memory function of the VACF (see insert of Fig. 3) we find for the hydrogen atoms [12]

$$
\Omega_{H}^{2}=11881 p s^{-2} \text {. }
$$

This value is to be compared to

$$
\Omega_{O}^{2}=1941 p s^{-2}
$$

for the oxygen atoms. Both the VACFs of hydrogen and oxygen have been obtained from a $100 \mathrm{ps}$ MD trajectory using autoregressive modeling [25,26] with 400 poles and a sampling time step of $10 \mathrm{fs}$.

As for the atomic mean square velocity we use the exact value,

$$
\left\langle v^{2}\right\rangle=\frac{k_{B} T}{M_{\mathrm{eff}}},
$$

where $M_{\text {eff }}$ is the effective mass of the atom under consideration, which has been determined according to expression (38). We consider the geometry of the SPC/E 


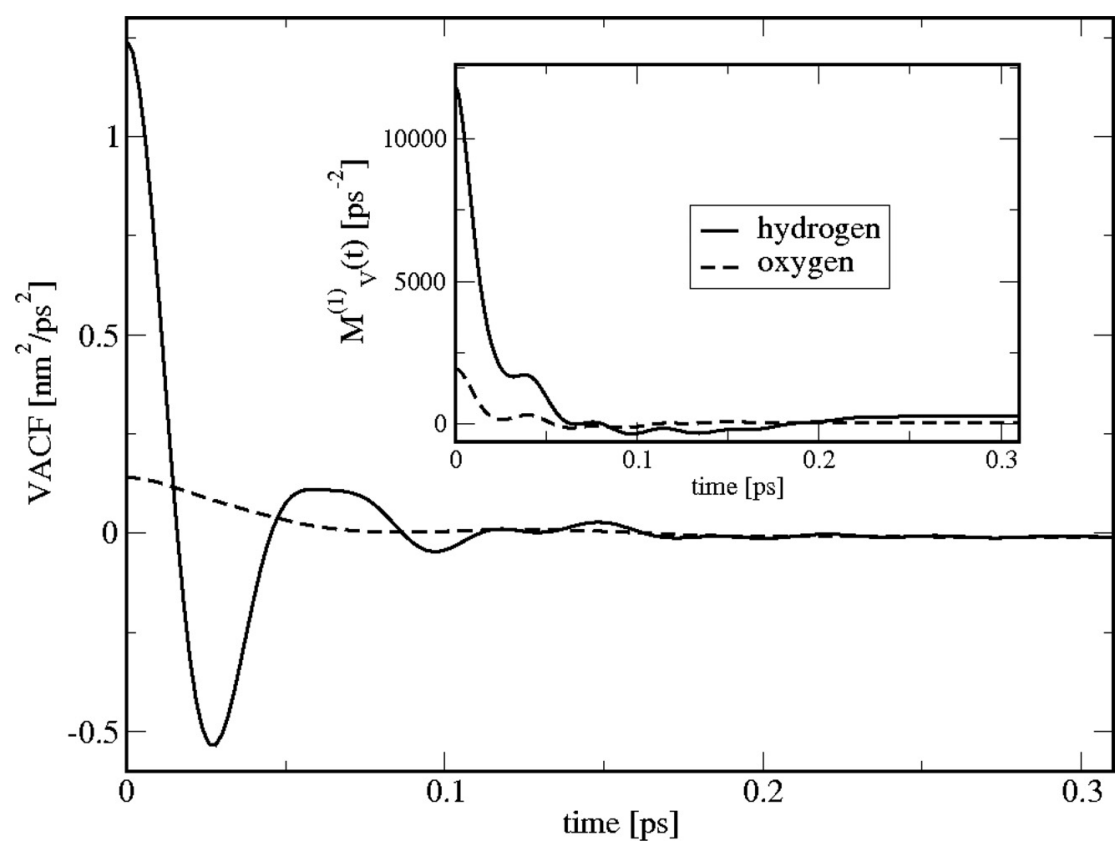

Fig. 3. VACF for hydrogen (solid line) and oxygen (broken line). The inset show the corresponding memory functions.

water model, where the water molecules are rigid, with fixed $O-H$ bond lengths of $1 \AA$ and with a fixed $H-O-H$ angle of $109.47^{\circ}$. The masses are $m_{H_{1}}=m_{H_{2}}=$ 1.008 and $m_{O}=16$, using atomic mass units. This leads to the three distance constraints,

$$
\begin{array}{r}
h_{1}(\mathbf{r}) \equiv\left(\mathbf{r}_{O}-\mathbf{r}_{H_{1}}\right)^{2}-1=0 \\
h_{2}(\mathbf{r}) \equiv\left(\mathbf{r}_{O}-\mathbf{r}_{H_{2}}\right)^{2}-1=0 \\
h_{3}(\mathbf{r}) \equiv\left(\mathbf{r}_{H_{1}}-\mathbf{r}_{H_{2}}\right)^{2}-8 / 3=0
\end{array}
$$

where all lengths are measured in $\AA$. Constructing $\mathbf{A}$ according to (27) and $\mathbf{M}$ according to (32), the pseudoinverse mass matrix $\mathbf{M}_{c}^{+}$can be obtained from expression (35). From the resulting matrix the block matrices $\mathbf{m}_{11}{ }^{+}, \mathbf{m}_{22}{ }^{+}$, and $\mathbf{m}_{33}{ }^{+}$can be extracted, and the effective masses are computed according to definition (38). Here 1,2,3 correspond, respectively, to $H_{1}, H_{2}$, and $O$. The result is [11]

$$
\begin{aligned}
m_{H_{1}} & =m_{H_{2}}=1.896, \\
m_{O} & =17.08 .
\end{aligned}
$$




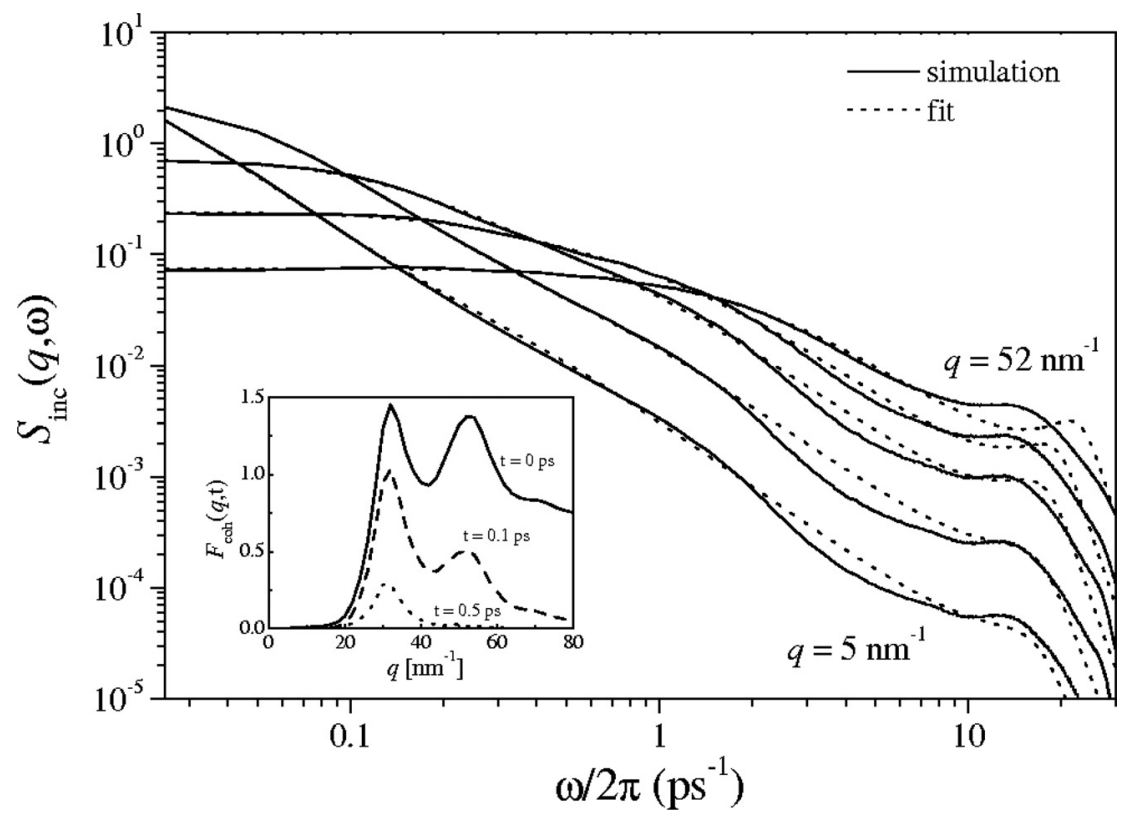

Fig. 4. Log-log plot of the incoherent dynamic structure factor for liquid water at ambient temperature and pressure from MD simulation (solid lines) and the corresponding fits of the analytical model (dotted lines) . The different curves correspond to (from bottom to top) $q=$ $5 \mathrm{~nm}^{-1}, q=10 \mathrm{~nm}^{-1}, q=20 \mathrm{~nm}^{-1}, q=32 \mathrm{~nm}^{-1}, q=52 \mathrm{~nm}^{-1}$, respectively. The parameters of the fits are given in Table 1. The inset shows the coherent intermediate scattering function as a function of $q$ for different times. Part of the data have been taken from Ref. [12].

The corresponding mean square velocities are $\left\langle v_{H}^{2}\right\rangle=1.315 \mathrm{~nm}^{2} / \mathrm{ps}^{2}$ and $\left\langle v_{O}{ }^{2}\right\rangle=0.146 \mathrm{~nm}^{2} / \mathrm{ps}^{2}$. These theoretical values may be compared to $\left\langle v_{H}{ }^{2}\right\rangle=$ $1.330 \mathrm{~nm}^{2} / \mathrm{ps}^{2}$ and $\left\langle v_{O}^{2}\right\rangle=0.147 \mathrm{~nm}^{2} / \mathrm{ps}^{2}$ which we obtained from the MD trajectories.

Taking $\left\langle v_{H}^{2}\right\rangle$ and $\Omega_{H}{ }^{2}$ as fixed parameters, we fitted the dynamic structure factor of the model to the simulated incoherent dynamic structure factor in order to obtain the model parameters $\alpha, \tau_{1}$ and $\tau_{2}$. The results are shown in Fig. 4 and Table 1 gives the respective fitted values for $\alpha, \tau_{1}$ and $\tau_{2}$. The fits have been performed at five different $q$-values, where $q=32 \mathrm{~nm}^{-1}$ and $q=52 \mathrm{~nm}^{-1}$ correspond to the maxima of the structure factor which is shown in the inset of Fig. 4. Fixing all parameters of the model to the values of the fit, we computed the corresponding intermediate scattering function, the first and second order memory functions, and the density of states. The results are displayed in Figs. 5, 6, and 7 , respectively, (broken lines), as compared to the corresponding simulation results (solid lines).

The dynamic structure factor of the model and the intermediate scattering function have been obtained from expression (14), using in the first case relation 


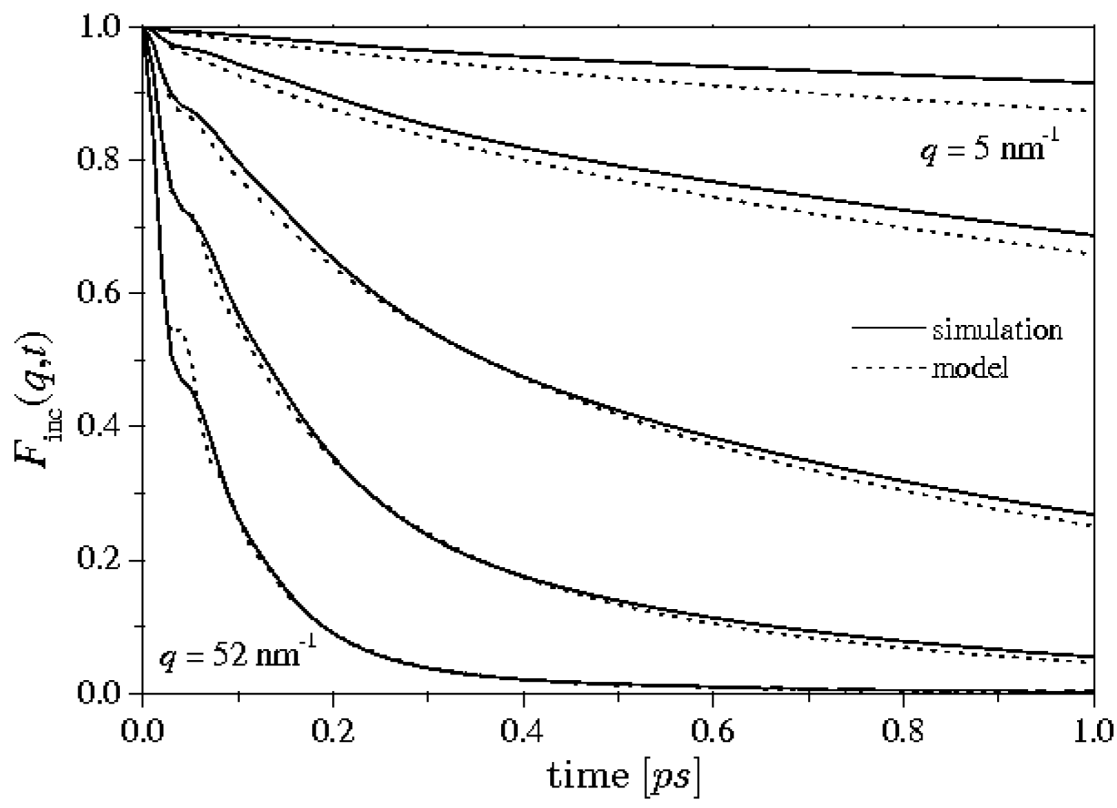

Fig. 5. Incoherent intermediate scattering function for water at ambient temperature and pressure from MD simulation (solid lines) and from the analytical model using the parameters given in Table 1 (dotted lines). The different curves correspond to (from top to bottom) $q=5 \mathrm{~nm}^{-1}, q=$ $10 \mathrm{~nm}^{-1}, q=20 \mathrm{~nm}^{-1}, q=32 \mathrm{~nm}^{-1}, q=52 \mathrm{~nm}^{-1}$, respectively.

(15) and performing in the second case an inverse Laplace transform. The model DOS has been obtained using the fit parameters corresponding to $q=5 \mathrm{~nm}^{-1}$ (see Table 1). In principle one should consider the limit $q \rightarrow 0$, but the latter limit cannot be obtained numerically, and one must resort to considering the smallest possible value for $q$, for which statistically meaningful results can be obtained from the simulation. From the size of the cubic simulation box, which is $L=1.9552 \mathrm{~nm}$, it follows that $q_{\min }=2 \pi / L \approx 3.2 \mathrm{~nm}^{-1}$ is the mathematically smallest possible value for $q$.

To test the validity of the model, we computed the intermediate scattering functions and the corresponding first and second order memory functions by the method described in Section 3, using a short MD trajectory of $20 \mathrm{ps}$ and autoregressive modeling with 1000 poles and a sampling time step of $2 f s$. Fig. 5 shows that the incoherent intermediate scattering function is remarkably well reproduced by the model, and the same is true for the first order memory functions (left part of Fig. 6). Even the second order memory function (right part of Fig. 6) shows the correct limiting behavior. Here we recall that the model parameters have been obtained from a fit to the simulated dynamic structure factor only. 


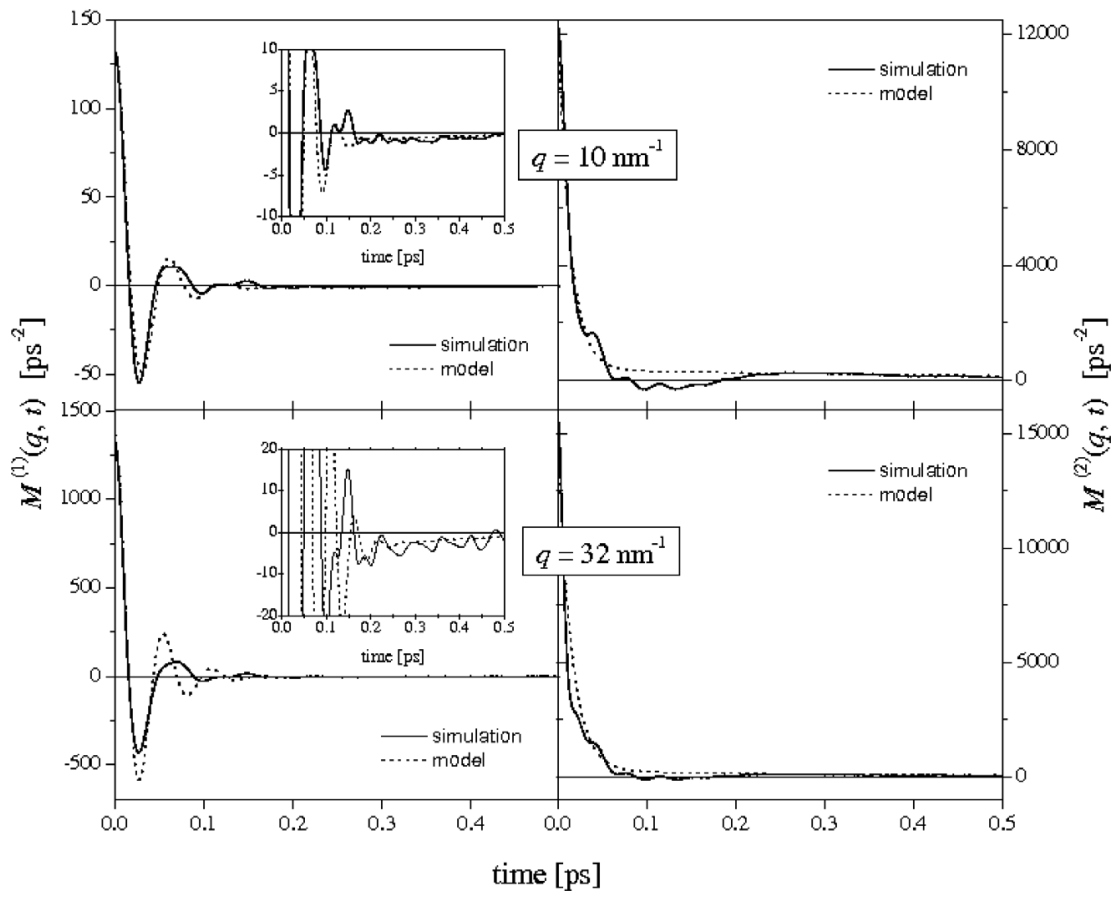

Fig. 6. First order and second order memory functions of the incoherent intermediate scattering function for water at ambient temperature and pressure for $q=10 \mathrm{~nm}^{-1}$ and $q=32 \mathrm{~nm}^{-1}$, as obtained from AR analysis of MD trajectories (solid lines) and from the analytical model using the parameters given in Table 1 (dotted lines). The insets show zooms on the corresponding functions. Part of the data have been taken from Ref. [12].

It should be noted that the quality of the agreement between model and simulation is reduced for higher frequencies with $\omega / 2 \pi \geq 3 \mathrm{THz}$. We attribute this finding to the fact that the fit yields two real and two complex conjugate poles for $\hat{F}_{s}(q, s)$. The whole inelastic spectrum is thus described by only two poles and one cannot expect more than a semi-quantitative agreement for the inelastic part of the dynamic structure factor and the density of states.

\subsection{Diffusion coefficient}

From the fitted parameters of the model one may also compute the diffusion coefficient according to Eq. (18). Using the fit parameters of Table 1 for $q=$ $5 \mathrm{~nm}^{-1}$ and $\Omega_{H}{ }^{2}=11881 \mathrm{ps}^{-2}$, one obtains $D=3.28 \cdot 10^{-5} \mathrm{~cm}^{2} / \mathrm{s}$, which is somewhat above the reference value of $D=2.55 \cdot 10^{-5} \mathrm{~cm}^{2} / \mathrm{s}$ obtained from the slope of the simulated mean square displacement of the hydrogen atoms. Here we used that $\lim _{q \rightarrow 0} F_{s}(q, t)=\exp \left(-D q^{2} t\right)$, such that the diffusion coefficient of the model should tend to the translational diffusion coefficient in the limit $q \rightarrow$ 


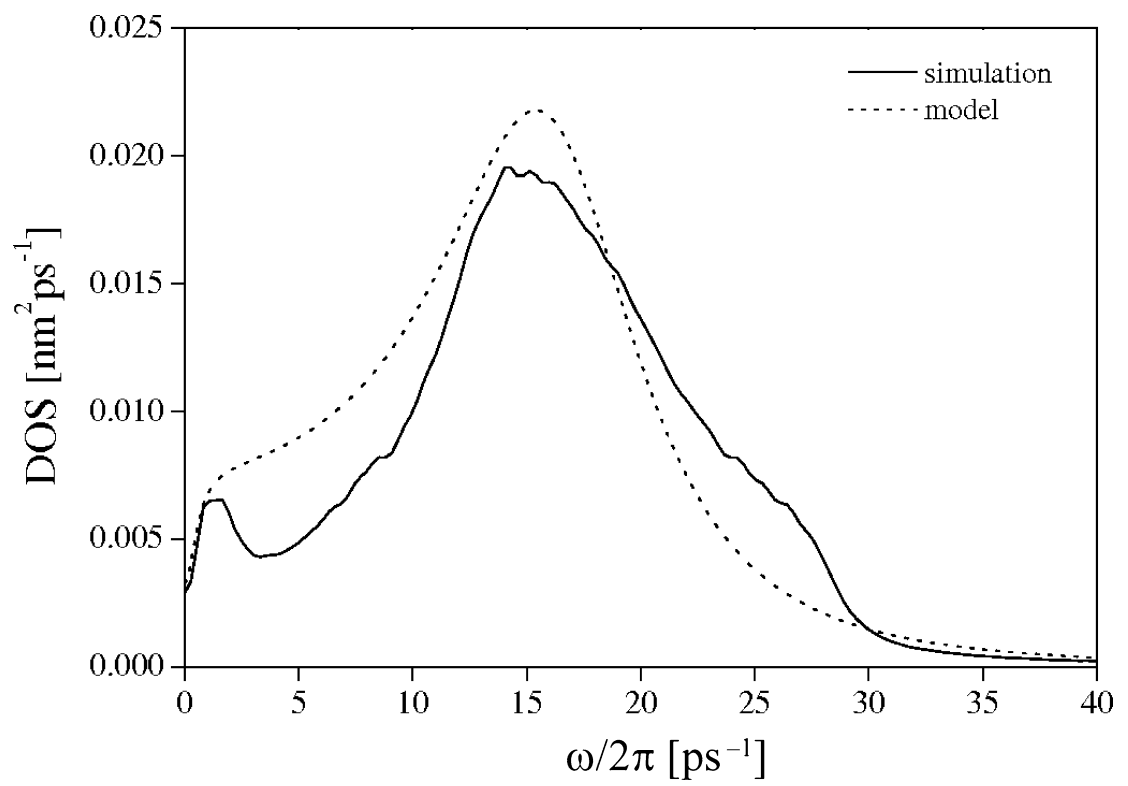

Fig. 7. Fourier transform of the average velocity autocorrelation function of the hydrogen atom in water at ambient temperature and pressure obtained from MD simulation (solid line) and from the analytical model using the parameters given in Table 1 (dotted line). The data have been taken from Ref. [12].

Table 1. Fitted values for the parameters of the analytical model. The parameters have been obtained from fits to the simulated dynamic structure factor shown in Fig. 1. The data have been taken from Ref. [12].

\begin{tabular}{lccc}
\hline$q\left(\mathrm{~nm}^{-1}\right)$ & $\alpha$ & $\tau_{1}(\mathrm{ps})$ & $\tau_{2}(\mathrm{ps})$ \\
\hline 5 & $0.9840(3)$ & $0.0139(3)$ & $1.08(2)$ \\
10 & $0.969(2)$ & $0.0141(8)$ & $0.66(2)$ \\
20 & $0.973(3)$ & $0.016(1)$ & $0.57(3)$ \\
32 & $0.981(3)$ & $0.0154(7)$ & $0.41(5)$ \\
52 & $0.99(1)$ & $0.013(1)$ & $0.2(2)$ \\
\hline
\end{tabular}

0 . The value of $q=5 \mathrm{~nm}^{-1}$ was again used as an approximation for the limit $q$ $\rightarrow 0$.

It should be noted that the diffusion coefficient obtained from the model is close to the one obtained from the relation $\gamma \approx D q^{2}$, where $\gamma$ is the friction constant defined in Eq. (3), using here the memory function of hydrogen. Alternatively one may use relation (19) and write $D=\left\langle v^{2}\right\rangle / \gamma_{v}$, where $\gamma_{v}$ is the integral over the first order memory function of the VACF. Using the same parameters as for the calculation of the diffusion coefficient, one finds $\gamma_{v}=$ $367.8 \mathrm{ps}^{-1}$ for the friction constant of the model. The latter may be compared to 
the result of $\gamma_{v}=349.8 \mathrm{ps}^{-1}$, which is obtained from a numerical integral over the memory function of the VACF calculated from the MD trajectory. To estimate the VACF we used in the latter case an autoregressive model of order $P=$ 1000 with a sampling time step of $\Delta t=0.001 \mathrm{ps}$ and the fact that the friction constant can be obtained via

$$
\gamma_{v}=\Delta t M_{v,>}^{(1)}(1)
$$

using expression (3) with $n=1$ and $M^{(0)}>(z)=c_{>}(z) \equiv c_{v v,>}(z)$. If the numerical value of $\gamma_{v}=349.8 \mathrm{ps}^{-1}$ is inserted into relation (19), one obtains $D=3.76$. $10^{-5} \mathrm{~cm}^{2} / \mathrm{s}$.

\section{Conclusion}

In this article we have shown how theory and simulation may be combined to develop simple but realistic models for the dynamics of liquid water. Using indepth analyses of MD trajectories of liquid water we were able to show that the validity of an existing model for the center-of-mass dynamics can be considerably extended to describe not only the diffusive motions seen in quasielastic neutron scattering experiments, but also faster motions, such as intermolecular vibrations and molecular librations. The point of departure was a Mori-Zwanzig type model for the intermediate self scattering function, in which the corresponding second order memory function is represented by a weighted sum of two exponentials. We have shown how this model can be extended to describe also the atomic dynamics in liquids consisting of rigid or semi-rigid molecules. This extension concerns the mean square velocity, for which an analytical expression can be derived from the molecular geometry, and the so-called Einstein frequency, which is the initial value of the memory function associated with the velocity autocorrelation function. For the latter quantity we have derived a formal expression, which is, however, yet difficult to handle in practice. For this reason we used the numerical values of the Einstein frequencies for oxygen and hydrogen obtained from the MD simulations.

An important technical point in our study was the use of an improved numerical method for the calculation of memory functions, which yields better numerical stability compared to earlier work, and enables the calculation of higher order memory functions in a particularly simple way. We applied the method in particular to compute the first and second order memory functions for the incoherent intermediate scattering function, which are the pivot elements in the analytical model discussed in this article.

Our results demonstrate that the intermediate self-scattering function of the hydrogen atoms, the corresponding dynamic structure factor, and the associated first order memory function are well reproduced by the analytical model if appropriate values are used for mean square velocity and the Einstein frequency. It should be stressed that the simple bi-exponential form of the second order mem- 
ory function yields the correct limiting behavior of its numerical counterpart for small and long times.

As for the slow diffusive dynamics, we have shown that the integrals over the memory functions for the incoherent intermediate scattering function of hydrogen and oxygen, respectively, have very similar values, although the form of the memory functions differs considerably. Since it is essentially the integral of the memory function which determines the slow relaxation and diffusion dynamics in liquids, this finding is consistent with the fact that the long time diffusion coefficient for different reference points in a rigid molecule is the same. The different form of the memory functions for hydrogen and oxygen atoms in a water molecule is essentially reflected in the different short time behavior of the corresponding intermediate scattering functions.

We have also shown that the model diffusion coefficient obtained from the fit parameters is somewhat overestimated, as compared to the value obtained from the slope of the simulated mean square displacement of the hydrogen atoms, which is close to the experimental value obtained by NMR measurements.

\section{References}

1. A. Rahman, Phys. Rev. 136(2A) (1964) 405-411.

2. J. P. Boon and S. Yip, Molecular Hydrodynamics. McGraw Hill, New York (1980).

3. J.-P. Hansen and I.R. McDonald, Theory of simple liquids. 2nd edition, Academic Press (1986).

4. U. Balucani and M. Zoppi, Dynamics of the liquid state. Clarendon Press, Oxford (1994).

5. R. Zwanzig, Statistical mechanics of irreversibility. pages 106-141. Lectures in Theoretical Physics. Wiley-Interscience, New York, (1961).

6. H. Mori, Progress of Theoretical Physics 33 (1965) 423-455.

7. H. Mori, Progress of Theoretical Physics 34(3) (1965) 399-416.

8. R. Zwanzig, Nonequilibrium statistical mechanics. Oxford University Press (2001).

9. B. J. Berne, J. P. Boon, and S. A. Rice, J. Chem. Phys. 45 (1966) 1086-1096.

10. R. G. Sachs and E. Teller, Phys. Rev. 60 (1941) 18-27.

11. G. R. Kneller, J. Chem. Phys. 125 (2006) 114107.

12. V. Calandrini, G. Sutmann, A. Deriu, and G. R. Kneller, J. Chem. Phys. 125 (2006) 236102.

13. V. Calandrini, A. Deriu, G. Onori, R. E. Lechner, and J. Pieper, J. Chem. Phys 120 (2004) 4759-4767.

14. V. Calandrini, A. Deriu, G. Onori, A. Paciaroni, and M. T. F. Telling, J. Phys. Cond. Matter (2006).

15. D. Di Cola, A. Deriu, and M. Sampoli, Physica B 226 (1996) 46-50.

16. M. Di Bari, A. Deriu, and M. Sampoli, Physica B 266(1-2) (1999) 92-96.

17. V. Calandrini, A. Deriu, G. Onori, R. E. Lechner, and J. Pieper, Appl. Phys. A 74 (2002) 1339-1341.

18. G. Ciccotti, M. Ferrario, and J. P. Ryckaert, Mol. Phys. 47(6) (1982) 1253-1264.

19. A. Ben-Israel and T. N. E. Greville, Generalized Inverses: Theory and Applications. John Wiley, New York (1974).

20. S. L. Campbell and C. D. Meyer (Jr), Generalized inverses of Linear Transformations. Pitman (1979).

21. G. H. Golub and C. F. Van Loan Matrix Computations. The Johns Hopkins University Press, Baltimore (USA), 3rd edition (1996). 
22. G. R. Kneller, J. Chem. Phys. 127 (2007) 164114.

23. H. J. C. Berendsen, J. R. Grigera, and T. P. Straatsma, J. Phys. Chem. 91 (1987) $6269-6271$.

24. W. Smith, C. W. Yong, and P.M. Rodger, Molecular Simulation 28(1) (2002) 385471.

25. A. Papoulis, Probablity, Random Variables, and Stochastic Processes. McGraw Hill, New York, 3rd edition (1991).

26. G. R. Kneller and K. Hinsen, J. Chem. Phys. 115(24) (2001) 11097-11105.

27. K. T. Gillen, D. C. Douglas, and M. J. R. Hoch, Journal of Chemical Physics 57 (1972) 5117. 